Check for updates

Cite this: RSC Adv., 2019, 9, 12737

Received 15th January 2019

Accepted 1st April 2019

DOI: $10.1039 / c 9 r a 00343 f$

rsc.li/rsc-advances

\section{The preparation of synthetic graphite materials with hierarchical pores from lignite by one-step impregnation and their characterization as dye absorbents $\dagger$}

\begin{abstract}
Tian Qiu, (DD ab Jian-Guo Yang, ${ }^{\text {*ab }}$ Xue-Jie Bai ${ }^{\mathrm{b}}$ and Yu-Ling Wang ${ }^{\mathrm{b}}$
Herein, synthetic graphite materials with hierarchical pores and large specific surface area were successfully prepared by one-step impregnation with lignite as the carbon source, sulfuric acid $\left(\mathrm{H}_{2} \mathrm{SO}_{4}\right)$ as the oxidant, and phosphoric acid $\left(\mathrm{H}_{3} \mathrm{PO}_{4}\right)$ as the activator. The microstructural characteristics of synthetic graphite were investigated via X-ray diffraction (XRD), Raman spectroscopy, scanning electron microscopy (SEM), and transmission electron microscopy (TEM). Moreover, the pore parameters were studied by nitrogen adsorption-desorption. The results showed that synthetic graphite had a perfect orderly layered structure with high graphitization degree and a well-developed multistage pore structure with pore sizes ranging from nanometer to micrometer. The specific surface area and pore volume were $415.29 \mathrm{~m}^{2} \mathrm{~g}^{-1}$ and $0.67 \mathrm{~cm}^{3} \mathrm{~g}^{-1}$, respectively. The results of Fourier transform infrared spectroscopy (FTIR) and X-ray photoelectron spectroscopy (XPS) showed that the impregnation pretreatment provided polar groups containing oxygen to the surfaces. These unique characteristics make synthetic graphite possess good adsorption capacity for dye pollutants (the adsorption rate of the methyl orange solution was $99.9 \%$ within $60 \mathrm{~min}$ at $50{ }^{\circ} \mathrm{C}$, and the $\mathrm{pH}$ value of the solution was 3). The effects of temperature and $\mathrm{pH}$ value on the adsorption capacity were studied. The repeatability of the adsorption performance was also tested, and the adsorption rate was $84.6 \%$ of the initial adsorption rate after five cycles.
\end{abstract}

\section{Introduction}

With the continuous improvement of the degree of industrialization in the world, a series of pollution problems have been brought about, among which water pollution has become a major problem affecting the human living environment. ${ }^{1}$ In particular, the wastewater discharged from the printing and dyeing industry is seriously polluting the water environment. Wastewater is characterized by difficult degradation, high chromaticity and high content of organic poisons, ${ }^{2-4}$ which may cause environmental pollution or direct harm to human health if it flows directly into rivers and seas. Therefore, how to detect and deal with industrial wastewater efficiently and reasonably has become a hot research topic. $^{5-9}$ The common wastewater treatment methods include chemical precipitation, redox reactions, biological metabolism and adsorption; ${ }^{\mathbf{1 0 - 1 5}}$ among them, adsorption has become one of the most potential methods for controlling water pollution

${ }^{a}$ National Engineering Research Center of Coal Preparation and Purification, China University of Mining and Technology, Xuzhou, Jiangsu 221116, China. E-mail: scetyjg@126.com

${ }^{b}$ School of Chemical Engineering and Technology, China University of Mining and Technology, Xuzhou, Jiangsu 221116, China

$\dagger$ Electronic supplementary information (ESI) available. See DOI: 10.1039/c9ra00343f because of its practicality, convenience, environmental friendliness and many other advantages. ${ }^{16-22}$

As a functional carbon material, expanded graphite has many superiorities such as multiple layers, abundant pore structures and large pore volume and specific surface area. Therefore, expanded graphite is an excellent adsorption material. ${ }^{23-26}$ In addition, expanded graphite is basically composed of pure carbon and is non-toxic; thus, it would not cause any secondary pollution to the environment. ${ }^{27}$ At present, to obtain expanded graphite, most researchers are employing natural flake graphite as a raw material, which is treated by an intercalation agent and oxidant before being expanded at high temperatures..$^{28-30}$ However, although there are abundant natural graphite resources in the world, the purity of raw graphite ore is relatively low; this makes the separation and purification process of graphite complex and costly. ${ }^{31}$ Therefore, it is necessary to find a cheaper raw material and a simpler preparation process for graphite with high adsorption property to substitute expanded graphite. In fact, coal-based graphite materials have been successfully prepared by several researchers and have been applied in many fields. ${ }^{32-36}$ However, most researchers employ high-rank coal, such as anthracite and bitumite, as raw materials to prepare coal-based graphite. Moreover, only few studies have been reported on the preparation of graphite from low-rank coal. 
As is well-known, lignite, a low-rank coal, is considered as a poor-quality energy source due to its high water content, high oxygen content and spontaneous combustion ability. ${ }^{37}$ However, its porous structure is one of the main characteristics that distinguishes lignite from high metamorphic coal, ${ }^{38,39}$ making lignite a very promising sorptive material. Due to the fact that researchers have successfully prepared activated carbon materials from lignite, ${ }^{\mathbf{4 0 - 4 2}}$ the preparation of porous graphite materials with large specific surface area from lignite is a very economical way.

In this study, lignite was used as a raw material for hightemperature graphitization heat treatment after impregnation with an oxidant $\left(\mathrm{H}_{2} \mathrm{SO}_{4}\right)$ and an activator $\left(\mathrm{H}_{3} \mathrm{PO}_{4}\right)$ to obtain synthetic graphite with a cellular microstructure. The microstructure and pore morphology have been discussed based on the microstructural analysis performed by a scanning electron microscope (SEM) and a transmission electron microscope (TEM). The degree of graphitization and the crystallinity parameter were characterized by X-ray diffraction (XRD) and Raman spectroscopy. The surface chemical composition of synthetic graphite materials was characterized by Fourier transform infrared spectroscopy (FTIR) and X-ray photoelectron spectroscopy (XPS). According to the adsorption characteristics, the specific surface area, pore volume and pore diameter distribution of synthetic graphite were obtained. Subsequently, the adsorption performance was investigated in an aqueous solution of methyl orange at different temperatures and $\mathrm{pH}$ values by an ultraviolet spectrophotometer (UV-vis).

\section{Experimental}

\subsection{Materials}

Sulfuric acid $\left(\mathrm{H}_{2} \mathrm{SO}_{4}\right)(\mathrm{AR})$, phosphoric acid $\left(\mathrm{H}_{3} \mathrm{PO}_{4}\right)(\mathrm{AR})$, sodium hydroxide $(\mathrm{NaOH})$ and sodium chloride $(\mathrm{NaCl})$ were purchased from Aladdin Industrial Corporation, Shanghai, China. Methyl orange was obtained from Beijing Chemical Reagent Company, China. Lignite, obtained from Inner Mongolia of China, was used as the starting precursor for the preparation of synthetic graphite materials. The raw coal was ground to powder and then sieved into a particle size of less than $74 \mu \mathrm{m}$. Lignite powder (LC), $\mathrm{H}_{2} \mathrm{SO}_{4}$ and $\mathrm{H}_{3} \mathrm{PO}_{4}$ were proportionally mixed ( $\mathrm{LC}(\mathrm{g}): \mathrm{H}_{2} \mathrm{SO}_{4}(\mathrm{~mL}): \mathrm{H}_{3}{ }^{-}$ $\left.\mathrm{PO}_{4}(\mathrm{~mL})=1: 0.1: 5\right)$. The mixture was stirred at $60{ }^{\circ} \mathrm{C}$ for 24 hours and filtered to obtain a sample of impregnated lignite (ILC). The proximate analysis and ultimate analysis results of LC and ILC are listed in Table 1.

Table 1 Proximate analysis and ultimate analysis results of the lignite sample ${ }^{a}$

\begin{tabular}{|c|c|c|c|c|c|c|c|c|}
\hline \multirow[b]{2}{*}{ Sample } & \multicolumn{3}{|c|}{$\begin{array}{l}\text { Proximate } \\
\text { analysis (wt } \%)\end{array}$} & \multicolumn{5}{|c|}{ Ultimate analysis (wt\%, daf) } \\
\hline & $M_{\mathrm{ad}}$ & $A_{\mathrm{d}}$ & $V_{\text {daf }}$ & $\mathrm{C}$ & $\mathrm{H}$ & $\mathrm{O}$ & $\mathrm{N}$ & $\mathrm{S}$ \\
\hline LC & 1.81 & 24.17 & 22.97 & 75.24 & 4.76 & 17.34 & 1.92 & 0.74 \\
\hline ILC & 1.26 & 3.05 & 19.41 & 79.39 & 4.51 & 13.86 & 1.25 & 0.99 \\
\hline
\end{tabular}

${ }^{a} M_{\text {ad }}:$ moisture (air dry basis); $A_{\mathrm{d}}:$ ash (dry basis); $V_{\text {daf }}$ : volatile (dry and ash-free basis); daf: dry and ash-free basis.
In the pickling process, most of the minerals in lignite were removed; thus, its ash content was significantly reduced. The removal of some hydrated minerals also resulted in a slight decrease in its moisture content. However, pickling does not have a significant impact on the carbon skeleton of lignite, and only a few active aliphatic hydrocarbon branched chains are removed by pickling; thus, there is no significant change in the volatile matter.

\subsection{Graphitization process}

The graphitization treatment process was carried out in a graphite furnace at high temperatures. During the graphitization process, the pressure in the furnace cavity was $40 \mathrm{kPa}$ higher than that in the normal atmosphere, and high purity argon $(99.99 \%)$ was used as the protective gas. The furnace was heated to the desired temperature (ranging from 1200 to 2700 ${ }^{\circ} \mathrm{C}$ ) at the heating rate of $20{ }^{\circ} \mathrm{C} \mathrm{min}^{-1}$ and maintained for 3 hours. The prepared synthetic graphite samples derived from impregnated lignite were denoted as ILG- $X$, where $X$ represented the graphitization temperature. Similarly, the synthetic graphite samples obtained by direct heat treatment of lignite without impregnation were defined as LG-X.

\subsection{Characterization of the synthetic graphite materials}

X-ray diffraction (D8 Advance, Bruker, Germany) with CuK $\alpha$ radiation was used for XRD measurements under the following conditions: $\lambda=0.1542 \mathrm{~nm}, 40 \mathrm{kV}$ tube voltage, $100 \mathrm{~mA}$ tube current, $50-60{ }^{\circ} \mathrm{C}$ temperature, and scanning range of $10-80^{\circ}$, and high-purity silicon used as an internal standard.

The interlayer spacing $\left(d_{002}\right)$ was determined using the Bragg's eqn (1), the graphitization degree $(g)$ was determined using the Franklin eqn (2), and the crystallite size $\left(L_{\mathrm{a}}\right)$ and the stacking height $\left(L_{\mathrm{c}}\right)$ were evaluated using the Scherrer formula (3). ${ }^{32}$

$$
\begin{gathered}
d_{(002)}=\lambda / 2 \sin \theta_{(002)} \\
g=\left[\left(0.3440-d_{(002)}\right) /(0.3440-0.3354)\right] \times 100 \% \\
L_{\mathrm{a}}=k_{1} \lambda /\left[\beta_{(100)} \cos \theta_{(100)}\right] \text { and } L_{\mathrm{c}}=k_{2} \lambda /\left[\beta_{(002)} \cos \theta_{(002)}\right]
\end{gathered}
$$

where $k$ is the Scherrer parameter $\left(k_{1}=1.84, k_{2}=0.94\right)$, $\beta$ represents the full width at half maximum (FWHM) of the diffraction peak, $\theta$ stands for the Bragg angle corresponding to the diffraction peak, and 0.3440 and 0.3354 are the carbon layer spacings $(\mathrm{nm})$ of original carbonaceous materials and ideal graphite crystal, respectively.

The morphological characteristics were investigated using SEM (Quanta 250, FEI, USA) and TEM (Tecnai G2 F20, FEI, USA). The Raman spectra were obtained by French JY LabRam HR800 under a $632.8 \mathrm{~nm}$ (He-Ne laser, $1.96 \mathrm{eV}$ ) laser excitation. The BET surface area and pore size distribution were measured using an auto $\mathrm{N}_{2}$ adsorption instrument (ASAP 2010, Micrometrics, USA). The specific surface area was calculated by the Brunauer-Emmett-Teller (BET) method. The total pore volume was calculated by the $\mathrm{N}_{2}$ adsorption amount at the relative pressure $P / P_{0}=0.99$. Moreover, the pore diameter distribution was calculated by the Barrett-Joyner-Halenda (BJH) method. 
The concentration of the dye was determined using a UV-visible recording spectrophotometer (UV-3100, Shimadzu Co. Japan). A Fourier transform infrared spectrometer (Vertex 80v, Bruker, Germany) was used for the characterization of polar groups on the surface of materials. X-ray photoelectron spectroscopy (XPS) was conducted using a multi-functional X-ray photoelectron spectrometer (ESCALAB 250Xi, Thermo Fisher, USA) with the pass energy of $29.35 \mathrm{eV}$ and a $\mathrm{MgK} \alpha$ line excitation source. The binding energy of C1s $(284.8 \mathrm{eV})$ was used as a reference.

\subsection{Adsorption and desorption experiments}

Methyl orange was used as the target adsorbent. The methyl orange solution $\left(0.1 \mathrm{~g} \mathrm{~L}^{-1}, 200 \mathrm{~mL}\right)$ mixed with a certain amount of the obtained synthetic graphite was added to a beaker, which was placed in a constant temperature water bath and continuously stirred. The $\mathrm{pH}$ value of the solution was adjusted by $20 \%$ $\mathrm{H}_{2} \mathrm{SO}_{4}$ and $20 \% \mathrm{NaOH}$. The $\mathrm{pH}$ of the adsorption system was measured by the PHS-3C meter (Shanghai Precision and Scientific Instrument Co. Ltd, China) at room temperature. The absorbance was measured by an ultraviolet spectrophotometer (UV-260, Shimadzu Co. Japan) after adsorption for a certain time followed by centrifugation and filtration.

$\mathrm{NaCl}$ dissolved in distilled water was used as the desorption solution. For each experiment, the desorption solution was taken at a certain concentration in a conical flask and placed in a constant temperature water bath oscillator. The $\mathrm{pH}$ value was adjusted, and certain amounts of samples to be desorbed were added. The concentration of methyl orange in the supernatant was determined every 10 min followed by centrifugation.

Calculation formula of the adsorption rate:

$$
W_{\mathrm{A}}=\left[\left(C_{0}-C_{\mathrm{at}}\right) / C_{0}\right] \times 100 \%=\left[\left(A_{0}-A_{\mathrm{at}}\right) / A_{0}\right] \times 100 \%
$$

Calculation formula of the desorption rate:

$$
W_{\mathrm{D}}=\left[C_{\mathrm{Dt}} /\left(C_{0}-C_{\mathrm{e}}\right)\right] \times 100 \%=\left[A_{\mathrm{Dt}} /\left(A_{0}-A_{\mathrm{e}}\right)\right] \times 100 \%
$$

where $C_{0}$ and $A_{0}$ represent the initial concentration and absorbance of the methyl orange solution at $462 \mathrm{~nm}$, corresponding to maximum absorption wavelength; $C_{\text {at }}$ and $A_{\text {at }}$ stand for the concentration and absorbance of methyl orange solution at $462 \mathrm{~nm}$ after a certain adsorption time; $C_{\mathrm{Dt}}$ and $A_{\mathrm{Dt}}$ are defined as the concentration and absorbance of the methyl orange solution at $462 \mathrm{~nm}$ after a certain desorption time; $C_{\mathrm{e}}$ and $A_{\mathrm{e}}$ are the adsorption equilibrium concentration and absorbance of the methyl orange solution at $462 \mathrm{~nm}$, corresponding to maximum absorption wavelength.

\section{Results and discussion}

\subsection{Microstructure characteristics of synthetic graphite materials}

The XRD patterns of synthetic graphite samples prepared at various graphitization temperatures from impregnated lignite are shown in Fig. 1. For the impregnated lignite, before heat treatment, the XRD pattern exhibits a broad and weak diffraction peak at about $26.3^{\circ}$, which corresponds to the reflection in

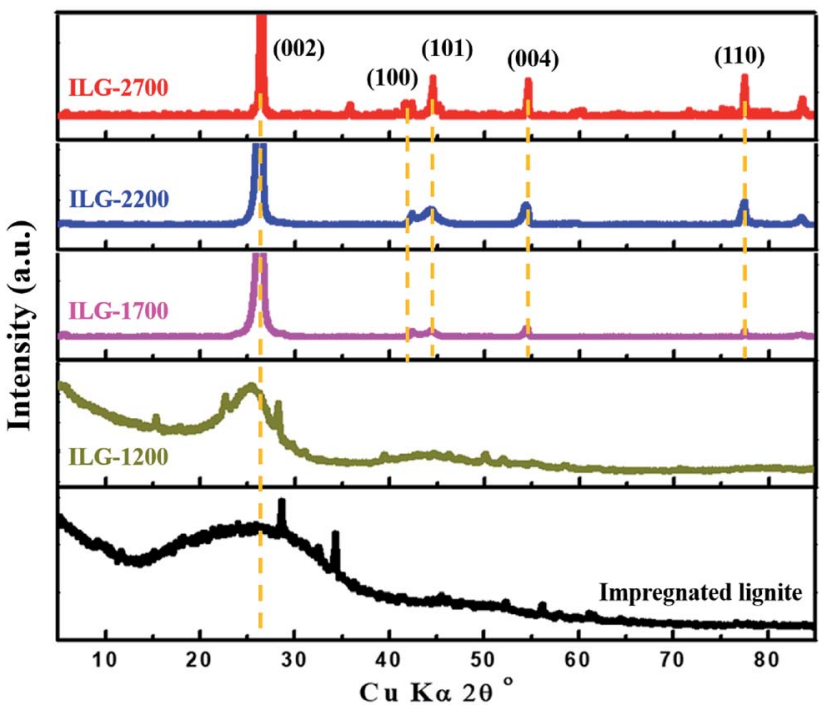

Fig. 1 XRD patterns of the impregnated lignite and synthetic graphite samples.

the (002) plane of aromatic layers, and a peak is barely visible at about $42.3^{\circ}$, which corresponds to the reflection in the (100) plane of aromatic layers. In the X-ray diffraction spectra of graphite, the (002) peak is attributed to the orientation of the aromatic ring carbon reticulated layers in three-dimensional arrangement; the (100) peak is attributed to the degree of condensation of the aromatic ring, that is, the size of the carbon mesh slice of the aromatic ring. The narrower and the higher the (002) peak, the better the orientation of the aromatic layer slice. The narrower and the higher the (100) peak, the larger the size of the aromatic layer slice. ${ }^{43}$ Based on the abovementioned theory and the XRD results, it is shown that there are some very small graphite microcrystalline structures in the impregnated lignite. Note that the ash content of lignite is greatly reduced after impregnation; however, the XRD patterns of LG-2700 and ILG-2700 show that there is no apparent difference among them (Fig. S1, Table S1 and more related discussion in ESI $\dagger$ ); this is one of the purposes of impregnation. After graphitization at the heat treatment temperature of $1200{ }^{\circ} \mathrm{C}$ on the impregnated lignite, the intensities of the (002) and (100) diffraction peaks are much higher than those in the case of impregnated lignite; this indicates that the stacking structure of the aromatic layers in the carbonized coal is enhanced. This enhancement is attributed to the decomposition of various aliphatic chains; moreover, the degree of aromatic ring condensation increases during the heat treatment process and then causes the twodimensional microcrystalline to grow gradually along the $a$ and $c$ axes. Note that the (002) and (100) diffraction peaks start to become sharp, and the diffraction peaks corresponding to the (101), (004) and (110) planes of graphite carbon ${ }^{44}$ start to emerge when the temperature reaches $1700{ }^{\circ} \mathrm{C}$. In addition, the diffraction peaks constantly shifted to higher angles as the temperature increased. Furthermore, the peaks grew narrower, and their intensity became higher; this indicated that the number of parallel carbon layers increased, and the aromatic 
Table 2 Structural parameters of synthetic graphite at different heat treatment temperatures

\begin{tabular}{lllrr}
\hline Sample & $d_{002}(\mathrm{~nm})$ & $g(\%)$ & $L_{\mathrm{a}}(\mathrm{nm})$ & $L_{\mathrm{c}}(\mathrm{nm})$ \\
\hline ILG-1700 & 0.3381 & 68.6 & 9.74 & 19.05 \\
ILG-2200 & 0.3374 & 76.7 & 16.29 & 21.30 \\
ILG-2700 & 0.3369 & 82.6 & 178.56 & 110.43
\end{tabular}

carbon grid layer became orderly arranged. Hence, it can be estimated that the graphite microcrystalline layer spacing $\left(d_{002}\right)$ is getting smaller. The structural parameters $g, L_{\mathrm{a}}$ and $L_{\mathrm{c}}$ of the microcrystals can also be calculated according to the eqn (1)-(3) to further understand the graphite crystalline structure of the synthetic graphite materials. The results are listed in Table 2.

It is observed that the crystallite size and stacking height of synthetic graphite enhances gradually with an increase in the treatment temperature. The values of $L_{\mathrm{a}}$ and $L_{\mathrm{c}}$ are of the same order of magnitude, showing polycrystalline characteristics. As the graphitization temperature increased, the interlayer spacing of the synthetic graphite materials decreased. At $2700{ }^{\circ} \mathrm{C}$, the $d_{002}$ of synthetic graphite decreased to $0.3369 \mathrm{~nm}$, close to that of ideal graphite $(0.3354 \mathrm{~nm})$. Hence, the degree of graphitization for ILG-2700 is $82.56 \%$, comparable to that of other synthetic graphite samples. ${ }^{36,45}$

Fig. 2 shows the Raman spectra of the synthetic graphite samples. For the vast majority of carbon materials, there are two peaks in the first-order scattering of Raman spectrum: one stretches at around $1580 \mathrm{~cm}^{-1}$ and the other around $1350 \mathrm{~cm}^{-1}$. The former is usually called the $\mathrm{G}$ peak, which represents the ordered structure and defect-free graphite, whereas the latter is always named the D peak, which symbolizes a disordered structure and graphite with defects. The intensity ratio of the two peaks $\left(I_{\mathrm{D}} / I_{\mathrm{G}}\right)$ reflects the order degree of the crystal when compared with that of graphite. ${ }^{46}$

As shown in Fig. 2(a), the intensity of the D peak gradually decreases, whereas the intensity of the $G$ peak increases with an increase in temperature; this indicates that the structural defects of carbon gradually disappear and are replaced by perfect crystal lattices during the graphitization process. In addition to the variations in the peak intensity, as the temperature increased, a red shift and a blue shift occurred in the D peak and the G peak, respectively, both of which turned to be narrow and sharp. The main reason for the red or blue shift of the spectra was the changes in the chemical bonds (the migration of electron clouds caused by variation in the intermolecular bond force and distance). Therefore, it could be deduced from the Raman spectra that higher heat-treatment temperatures led to an increase in the intermolecular bond force of the coal-based graphite and a decrease in the spacing between carbon layers. Based on a theory proposed by Tuinstra and Koenig, ${ }^{47}$ the crystallite size $\left(L_{\mathrm{a}}\right)$ could also be determined by the equation: $L_{\mathrm{a}}=4.4 /\left(I_{\mathrm{D}} / I_{\mathrm{G}}\right)$, where $I_{\mathrm{D}}$ and $I_{\mathrm{G}}$ stand for the intensity of the $\mathrm{D}$ peak and $\mathrm{G}$ peak, respectively. The calculation results are shown in Fig. 2(c), which are highly in accordance with those of the XRD analysis.

As shown in Fig. 2(b), all synthetic graphite samples have a second-order Raman peak at around $2700 \mathrm{~cm}^{-1}$. When the second-order peak is a single peak, that is, when no obvious peak splitting occurs, it indicates that the synthetic graphite has developed a two-dimensional graphite lattice, but the development of a three-dimensional lattice is imperfect. When the second-order peak has a splitting phenomenon, it indicates that the three-dimensional graphite lattice has been developed, and
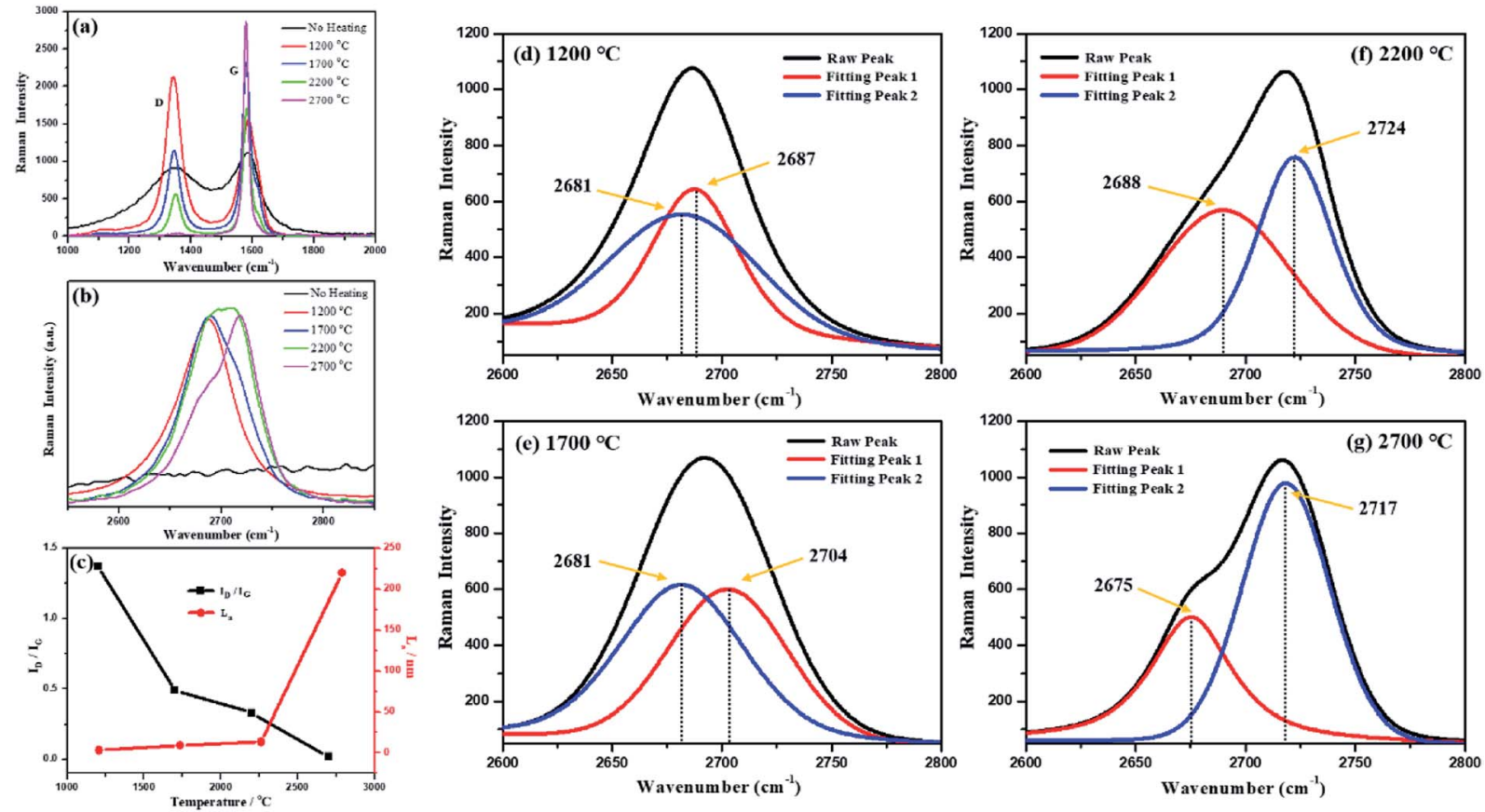

Fig. 2 Raman spectra of the synthetic graphite samples: (a) first-order Raman spectra; (b) second-order Raman spectra; (c) $I_{\mathrm{D}} / I_{\mathrm{G}}$ and the value of $L_{a} ;$ and $(d)-(g)$ peak-differentiating-imitating analysis of the second-order Raman spectra. 
the perfect degree of the three-dimensional graphite lattice development is proportional to the degree of spectral peak splitting and the intensity ratio of the splitting peaks. ${ }^{48}$ It can be seen from Fig. 2(d)-(g) that with an increase in the heat treatment temperature, the splitting degree of the second-order Raman peak and the ratio of the splitting peaks intensity gradually increase. This result further indicates that a highertemperature treatment is advantageous for the development of ordered graphite crystallites during the graphitization process. The Raman spectra of the graphitized products obtained without the impregnation pretreatment are shown in the ESI (Fig. S2†).

To demonstrate the effect of the impregnation treatment on the morphology of synthetic graphite samples, SEM analysis was carried out, and the corresponding images are shown in Fig. 3. It can be seen that there are many mineral components in raw coal (Fig. 3(a)). After impregnation (Fig. 3(b) and (c)), the minerals are removed, and many pores and cracks are left in the coal. After graphitization treatment at high temperatures, the synthesized graphite, i.e., both LG-2700 (Fig. 3(d)-(f)) and ILG2700 (Fig. 3(g)-(i)), display a highly ordered lamellar structure. Moreover, the orientation between the lamellae is different; this results in the development of some micron-sized pores. However, the difference is that the graphite sheet of LG-2700 is flat, and the surface is smooth without pores, whereas the graphite sheet of ILG-2700 is folded, which provides more secondary micropores for the material, and, more importantly, there are many defects and nanopores on the surface. These nanopores enable ILG-2700 to perform better in adsorption applications.

In the TEM images of ILG-2700 (Fig. 4), abundant and widespread layered graphite sheets can be found. Moreover, defects and nanopores are observed, as indicated in Fig. 4(c) and (d). The high-resolution TEM image shown in Fig. 4(d) clearly reveals that ILG- 2700 contains a highly ordered graphite crystalline structure mixed with a small amount of amorphous carbon. The diffraction had an annular pattern instead of a typical hexagonal speckle shape, and the diffraction rings of 002, 004, and 006 were clear and sharp, meaning that the ILG2700 belonged to polycrystalline graphite.

\subsection{Pore parameters of synthetic graphite materials}

For solid adsorbents, the interface is the main place where adsorption occurs, and the surface area is one of the important factors affecting the adsorption performance. Therefore, the pore parameter characteristics of the obtained synthetic graphite materials were further investigated by low-temperature nitrogen adsorption.

According to Fig. 5(a), the trends of the two isotherms are similar. The adsorption amount is less in the low-pressure area and increases sharply in the high-pressure area. In accordance with the IUPAC classification, the adsorption isotherms of LG-2700 and ILG-2700 belong to the type-III

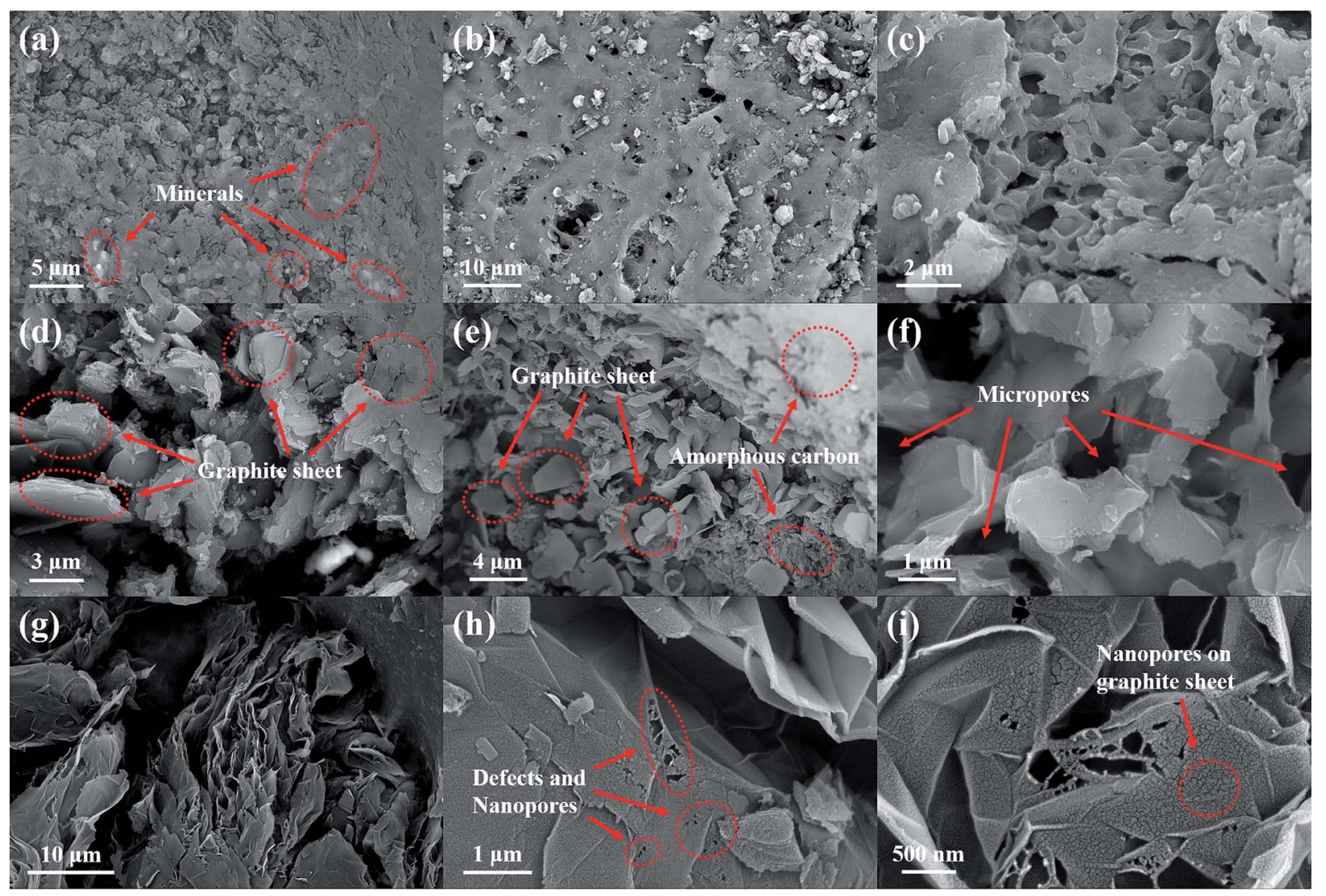

Fig. 3 SEM images of lignite, impregnated lignite and synthetic graphite samples: (a) lignite; (b) and (c) impregnated lignite; (d) - (f) LG-2700; (g)(i) ILG-2700. 


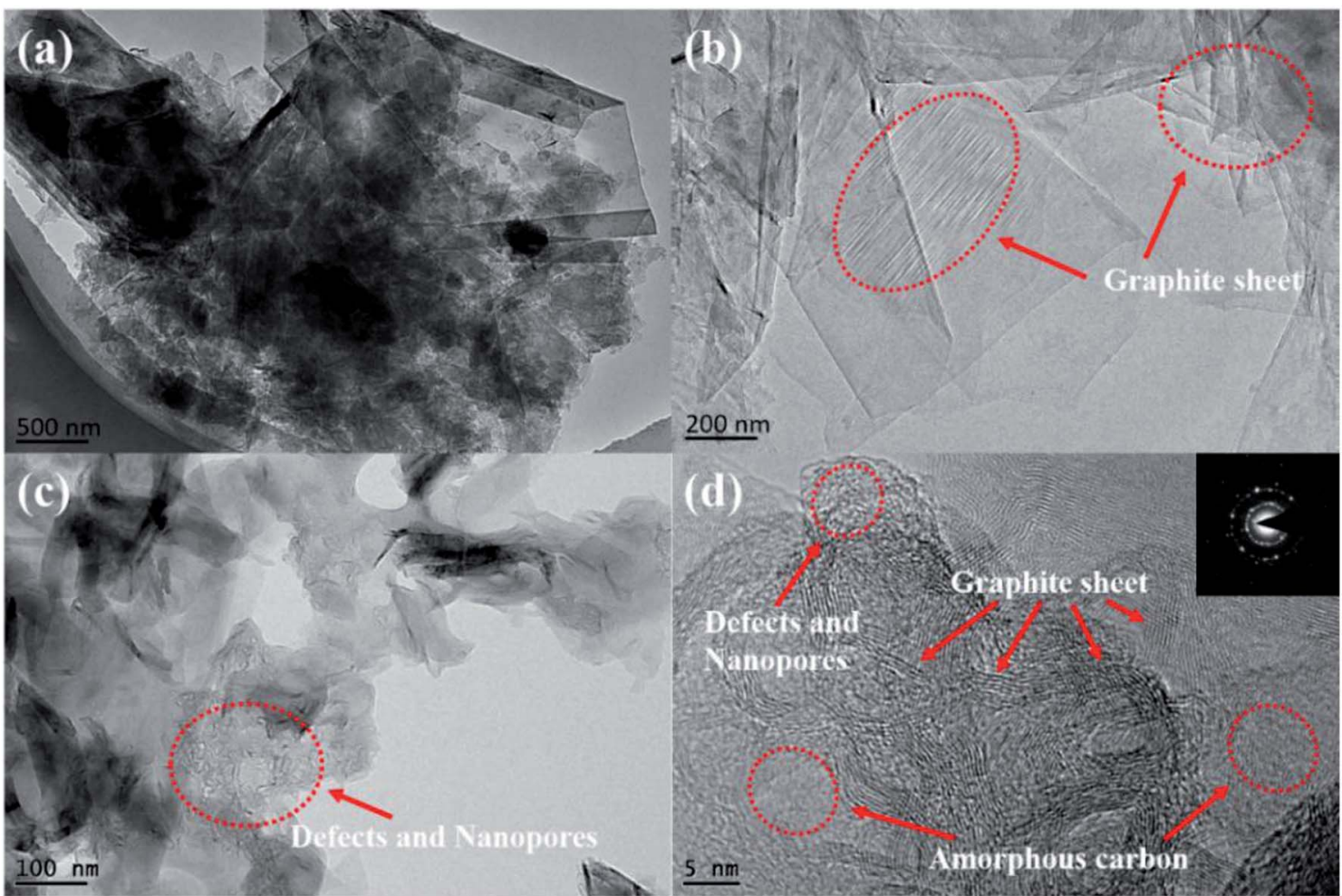

Fig. 4 TEM micrographs of synthetic graphite ILG-2700: (a), (b) and (c) TEM; (d) HRTEM and the diffraction pattern.
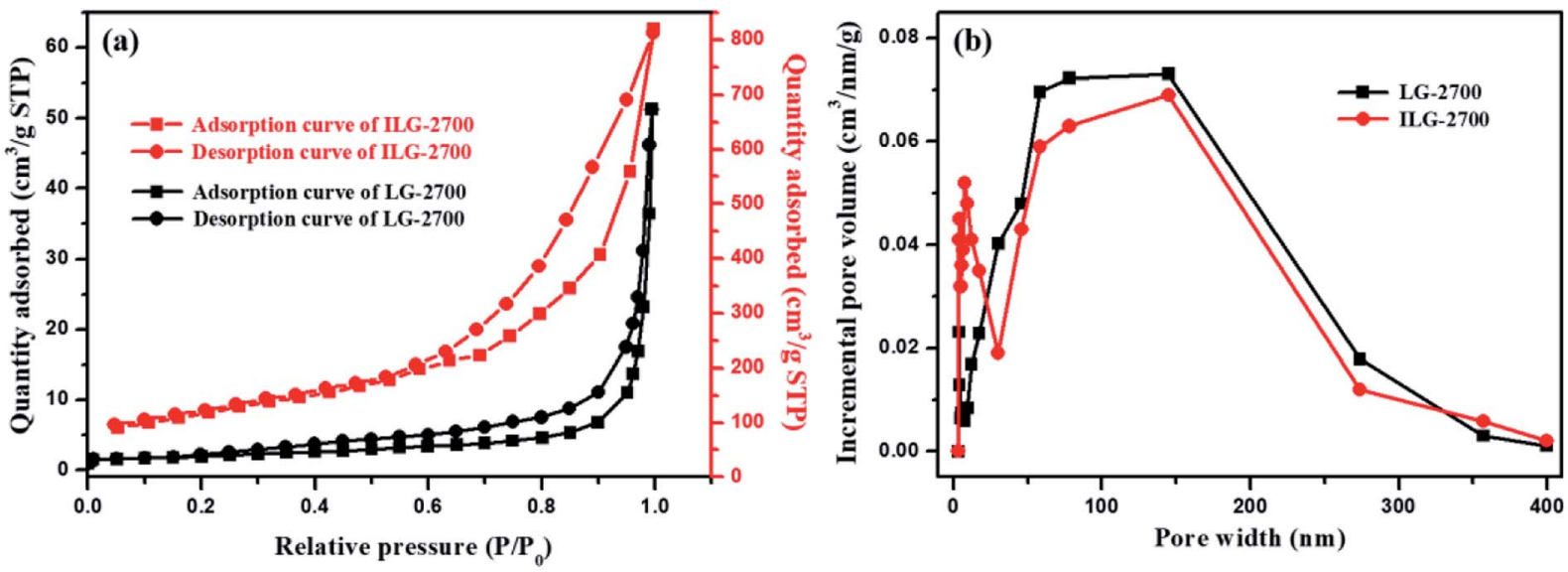

Fig. $5 \quad \mathrm{~N}_{2}$ adsorption-desorption isotherms (a) and pore size distribution (b) of LG-2700 and ILG-2700.

adsorption isotherm, and the hysteresis loop of ILG-2700 is type H3. This hysteresis loop is generated by the adsorption of a flaky material or a slit porous material, which is consistent with the morphology of the sample shown in the SEM images (see Fig. 3(g)). The adsorption isotherm of LG-2700 also showed adsorption hysteresis in the low-pressure area, but no hysteresis loop is formed. This may be due to the intercalated adsorption of nitrogen molecules on the graphite sheets and the difficulty of desorption in a low-pressure area. The BET specific surface area and total pore volume for LG-2700 and ILG-2700 are $7.03 \mathrm{~m}^{2} \mathrm{~g}^{-1}$ and $0.079 \mathrm{~cm}^{3} \mathrm{~g}^{-1}$ and $415.29 \mathrm{~m}^{2} \mathrm{~g}^{-1}$ and $0.67 \mathrm{~cm}^{3} \mathrm{~g}^{-1}$, respectively, which are much higher than those of anthracite-based graphite materials and natural graphite. ${ }^{32}$ The pore size distribution was evaluated by the BJH method (Fig. 5(b)). Compared with the case of LG-2700, the macropore in ILG-2700 is well developed, and some mesopores also appeared. This fully indicates that the impregnation process is of great help in the formation of a developed multistage pore structure. After impregnation, more new pore structures can be created, which lay the foundation for the improvement of the adsorption performance of LG-2700. 


\subsection{Surface chemical composition of synthetic graphite materials}

The surface polar groups of adsorbents favor the adsorption of dyes. To introduce more polar groups on the surfaces of the lignitebased synthetic graphite material, the material was treated with impregnation pretreatment. The FTIR technique was used for characterization (Fig. 6(a)). The absorbance peaks at about $3600 \mathrm{~cm}^{-1}$ and $1600 \mathrm{~cm}^{-1}$ correspond to the $-\mathrm{OH}$ stretching mode and - $\mathrm{CO}$ vibration mode of the keto form, respectively. It can be clearly seen that these peaks of ILG-2700 are significantly stronger than those of LG-2700. This indicates that the anticipated polar groups have been introduced on the surfaces of the lignite-based synthetic graphite material via the impregnation pretreatment.

Although there was no evident change in the XRD patterns of ILG-2700 and LG-2700 (see Fig. S1(b) †), it is clear via the XPS analysis that the surface oxygen element content increased, and the surface carbon element content decreased with the impregnation pretreatment (Fig. 6(b)). This also indicated that the polar groups containing oxygen were introduced; this was consistent with the FTIR analysis results.

\subsection{Adsorption and desorption performances of synthetic graphite materials}

The adsorption performances of the synthetic graphite ILG2700 were studied. The methyl orange solution $\left(0.1 \mathrm{~g} \mathrm{~L}^{-1}, 200\right.$ $\mathrm{mL}$ ) mixed with $0.1 \mathrm{~g}$ (the required amount of adsorbent was 5 $\times 10^{-4} \mathrm{~g} \mathrm{~mL}^{-1}$, which was much lower than that of coal fly ash $^{49}$ ) ILG-2700 was injected into the beaker, which was placed in a constant temperature water bath and continuously stirred. Fig. 7 shows the effect of the adsorption temperature and $\mathrm{pH}$ value on the adsorption rate. The adsorption rate between $20{ }^{\circ} \mathrm{C}$ and $50{ }^{\circ} \mathrm{C}$ increased with an increase in the temperature (Fig. 7(a)); this was attributed to the fact that the increase of temperature could effectively increase the migration rate of methyl orange, reduce the reaction activation energy and increase the number of activated molecules, which was the "solvent displacement" theory in liquid phase adsorption. ${ }^{\mathbf{5 0}}$ When the temperature is higher than $50{ }^{\circ} \mathrm{C}$, the vibration frequency of methyl orange at the active site increases; this results in an increase in the desorption rate and a decrease in the adsorption rate.
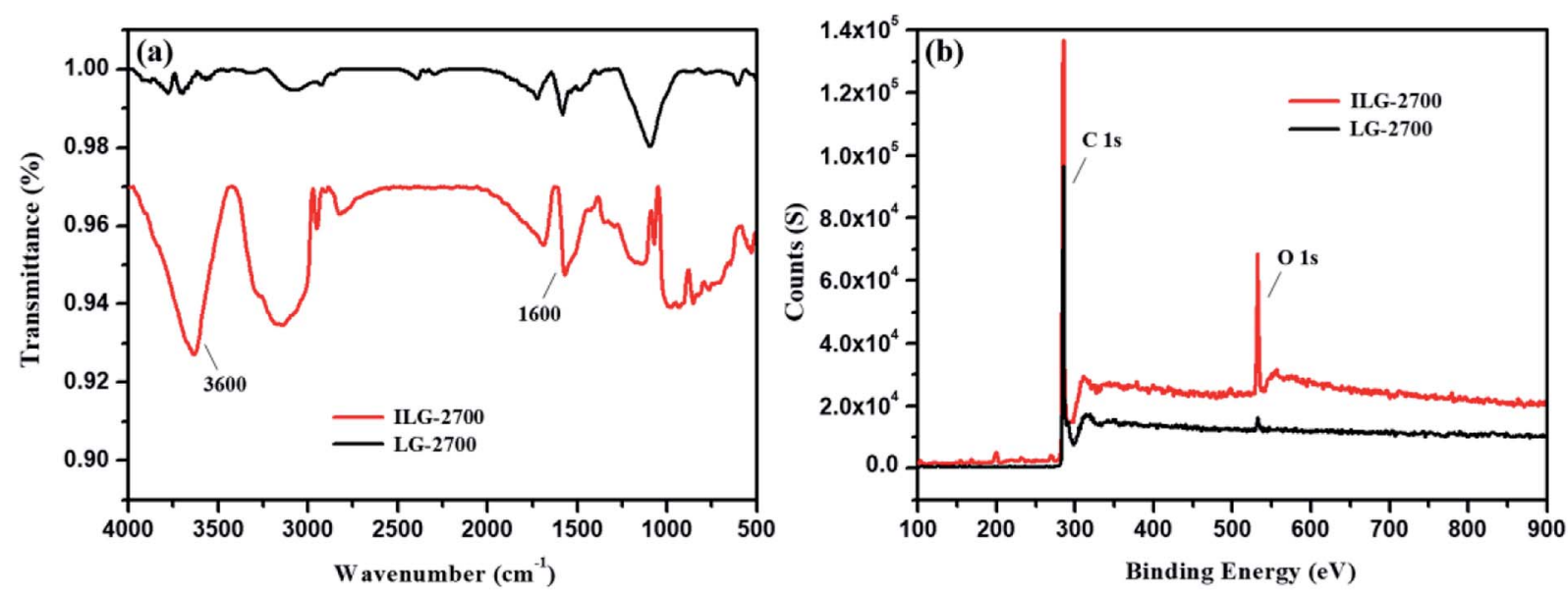

Fig. 6 FTIR spectra (a) and XPS survey spectra (b) of LG-2700 and ILG-2700.
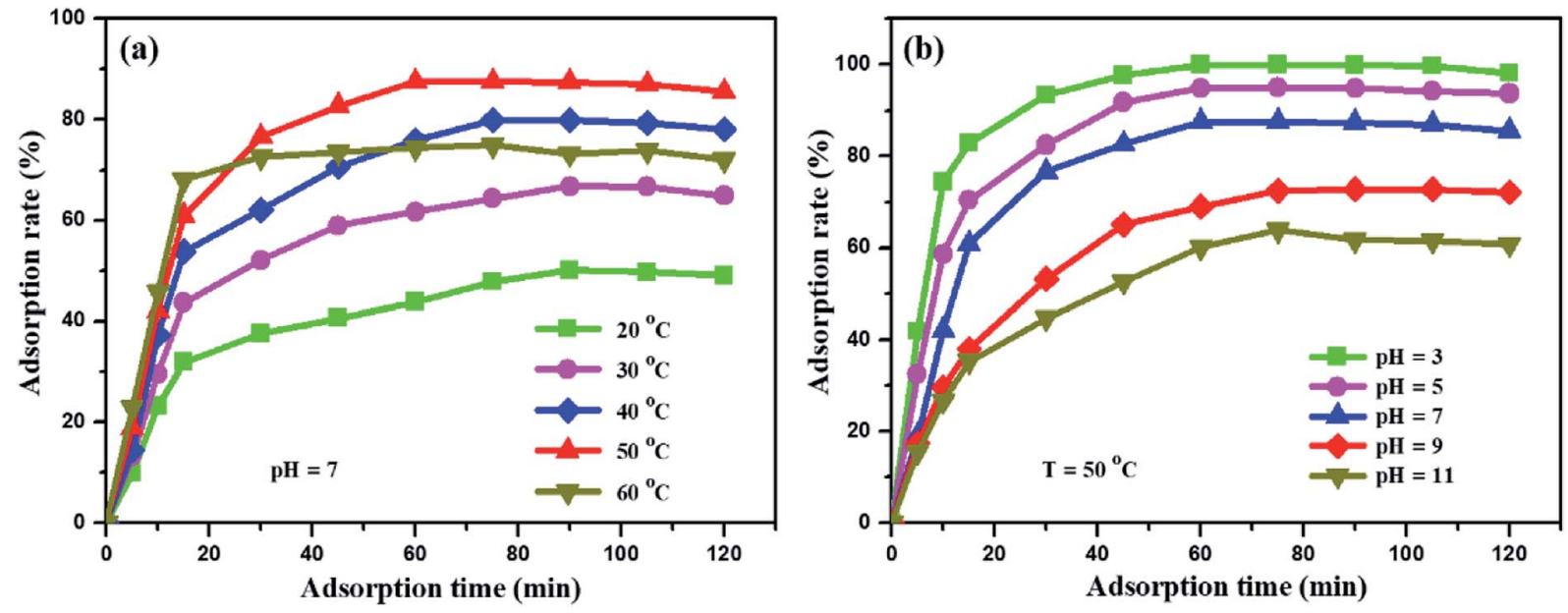

Fig. 7 Influence of adsorption temperature (a) and $\mathrm{pH}$ value (b) on the adsorption rate of the methyl orange solution. 
The form of methyl orange in the aqueous solution changes with a change in the $\mathrm{pH}$ value. ${ }^{51}$ Therefore, a change in the $\mathrm{pH}$ value will affect the adsorption mechanism and adsorption rate. In Fig. 7(b), it can be obviously seen that the adsorption rate is significantly improved under acidic conditions, and it increases from $64 \%$ to $99.9 \%$ at $50{ }^{\circ} \mathrm{C}$. Under acidic conditions, one end of the sulfonate is negatively charged, and methyl orange has a quinone structure (Fig. S3(a) $\dagger$ ), which can easily form chemical bonds; this will increase the adsorption of methyl orange to a certain extent. However, under alkaline conditions, methyl orange has an azobenzene structure (Fig. S3(b)†), which is not conducive to the adsorption of methyl orange by the synthetic graphite ILG-2700. When the adsorption equilibrium is reached for a period of time, the adsorption rate slightly decreases. It is estimated that the structure of expanded graphite is destroyed by long-time stirring; this leads to the desorption of methyl orange.

For adsorption materials, the purpose of desorption and regeneration is to reuse these materials. After repeated adsorption-desorption cycles, the desorption rate and the effect of repeated adsorption are important indicators for the performance of material recycling. In the desorption experiments, a $0.5 \mathrm{~mol} \mathrm{~L}^{-1} \mathrm{NaCl}$ solution with a $\mathrm{pH}$ value of 9 was chosen as the eluent (Fig. S4, discussion on the optimization process of eluents is provided in the ESI $\dagger$ ). The effect of multiple adsorption-desorption cycles of ILG-2700 for methyl orange is shown in Fig. 8. It can be seen from Fig. 8 that the adsorption rate of ILG- 2700 for methyl orange slightly decreases with an increase in cycle number. After 5 adsorption-desorption cycles, the adsorption rate remained above $84 \%$, and the desorption rate basically remained around $66 \%$. This indicates that ILG2700 has a better recycling performance and economic value compared with other adsorption materials. ${ }^{52-55}$ The gradual decrease in the adsorption capacity may be due to the fact that methyl orange without desorption occupies the adsorption site; this results in a slight decrease in subsequent adsorption or change in the ILG-2700 structure during desorption and regeneration. Therefore, further studies are required to improve the desorption rate to ensure that the surface of ILG-2700 after desorption has less residual methyl orange.

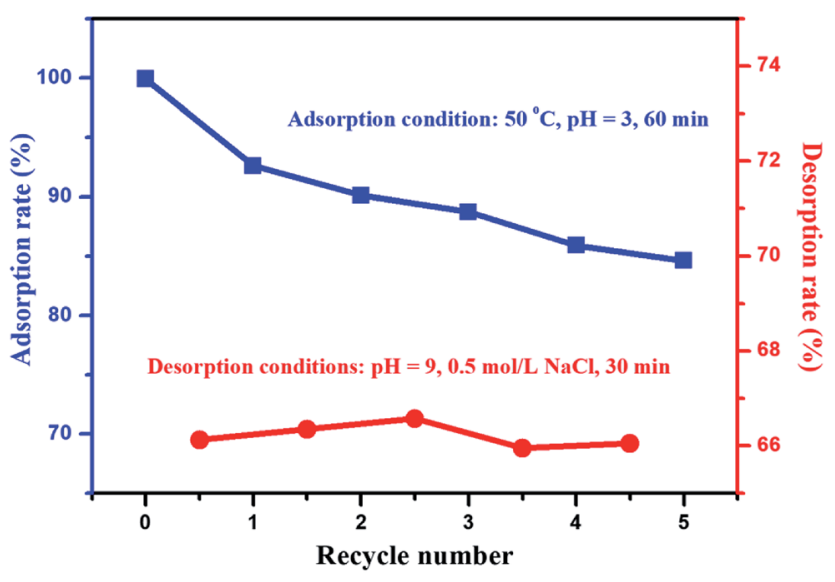

Fig. 8 Effect of the recycle number on adsorption and desorption.

\section{Conclusions}

In the present study, synthetic graphite materials with cellular microstructure meant to remove dye pollutants from water were successfully prepared via a simple, economical, and one-step impregnation method. XRD, Raman spectroscopy, SEM and TEM results show that the microstructures of synthetic graphite materials are strongly dependent on the graphitization temperature. The obtained synthetic graphite graphitized at $2700{ }^{\circ} \mathrm{C}$ (ILG-2700) has a perfect orderly layered structure with high graphitization degree and relatively large surface area with well-developed pores. Moreover, the impregnation pretreatment provides polar groups containing oxygen on the surfaces of ILG-2700. Due to these excellent characteristics, it is demonstrated that the synthetic graphite has outstanding adsorption performances such as a high adsorption rate $(99.9 \%$ within $60 \mathrm{~min}$ adsorption time). The $\mathrm{pH}$ value and temperature have a significant effect on the adsorption rate. The lignitebased graphite has a better ability to absorb methyl orange under the conditions of low $\mathrm{pH}$ value and higher temperature as compared to the conditions of high $\mathrm{pH}$ value and lower temperature. The regenerated adsorption has a good repeatability after five cycles.

\section{Conflicts of interest}

There are no conflicts to declare.

\section{Acknowledgements}

This work was supported by the National "Twelfth Five-Year" Plan for Science \& Technology (2014BAB01B02). The authors would like to thank the ShenHua Ningxia Coal Industry Group for financial support and providing Taixi anthracite samples. We also want to thank the Advanced Analysis \& Computation Center of China University of Mining and Technology for providing the support.

\section{References}

1 R. P. Schwarzenbach, T. Egli, T. B. Hofstetter, U. V. Gunten and B. Wehrli, Global Water Pollution and Human Health, Social Science Electronic Publishing, 2010, vol. 35.

2 Y. Sun, H. Zheng, M. Tan, Y. Wang, X. Tang, L. I. Feng and X. Xiang, Synthesis and Characterization of Composite Flocculant PAFS-CPAM for the Treatment of Textile Dye Wastewater, J. Appl. Polym. Sci., 2014, 131, 2113-2124.

3 M. A. Yun, K. M. Yeon, J. S. Park, C. H. Lee, J. Chun and D. J. Lim, Characterization of biofilm structure and its effect on membrane permeability in MBR for dye wastewater treatment, Water Res., 2006, 40, 45-52.

4 P. Banerjee, S. Sau, P. Das and A. Mukhopadhayay, Optimization and modelling of synthetic azo dye wastewater treatment using graphene oxide nanoplatelets: characterization toxicity evaluation and optimization using artificial neural network, Ecotoxicol. Environ. Saf., 2015, 119, $47-57$. 
5 S. Natarajan, H. C. Bajaj and R. J. Tayade, Recent advances based on the synergetic effect of adsorption for removal of dyes from waste water using photocatalytic process, $J$. Environ. Sci., 2018, 65, 201-222.

6 A. Salama and P. Hesemann, New N-guanidinium chitosan/ silica ionic microhybrids as efficient adsorbent for dye removal from waste water, Int. J. Biol. Macromol., 2018, 111, 762.

7 E. Gilpavas, I. Dobrosz-Gómez and M. Á. Gómez-García, Optimization of sequential chemical coagulation - electrooxidation process for the treatment of an industrial textile wastewater, Journal of Water Process Engineering, 2018, 22, 73-79.

8 A. A. El-Zahhar, N. S. Awwad and E. E. El-Katori, Removal of bromophenol blue dye from industrial waste water by synthesizing polymer-clay composite, J. Mol. Liq., 2014, 199, 454-461.

9 M. Wu, L. Sun, K. Miao, Y. Wu and L. J. Fan, Detection of Sudan Dyes Based on Inner Filter Effect with Reusable Conjugated Polymer Fibrous Membranes, ACS Appl. Mater. Interfaces, 2018, 10, 8287-8295.

10 M. S. Oncel, A. Muhcu, E. Demirbas and M. Kobya, A comparative study of chemical precipitation and electrocoagulation for treatment of coal acid drainage wastewater, J. Environ. Chem. Eng., 2013, 1, 989-995.

11 X. Vu, J. Y. Lin, Y. J. Shih and Y. H. Huang, Reclaiming Boron as Calcium Perborate Pellets from Synthetic Wastewater by Integrating Chemical Oxo-Precipitation within a FluidizedBed Crystallizer, ACS Sustainable Chem. Eng., 2018, 6, 4784-4792.

12 M. L. Daumer, F. Béline, F. Guiziou and M. Sperandio, Influence of $\mathrm{pH}$ and Biological Metabolism on Dissolved Phosphorus during Biological Treatment of Piggery Wastewater, Biosystems Engineering, 2007, 96, 379-386.

13 Z. Y. Sui, Y. Cui, J. H. Zhu and B. H. Han, Preparation of three-dimensional graphene oxide-polyethylenimine porous materials as dye and gas adsorbents, ACS Appl. Mater. Interfaces, 2013, 5, 9172-9179.

14 M. Feng, W. You, Z. Wu, Q. Chen and H. Zhan, Mildly alkaline preparation and methylene blue adsorption capacity of hierarchical flower-like sodium titanate, $A C S$ Appl. Mater. Interfaces, 2013, 5, 12654-12662.

15 L. Zhou, B. He and J. Huang, One-step synthesis of robust amine- and vinyl-capped magnetic iron oxide nanoparticles for polymer grafting, dye adsorption, and catalysis, ACS Appl. Mater. Interfaces, 2013, 5, 8678-8685.

16 A. Chen, Y. Li, Y. Yu, Y. Li, K. Xia, Y. Wang and S. Li, Synthesis of mesoporous carbon nanospheres for highly efficient adsorption of bulky dye molecules, J. Mater. Sci., 2016, 51, 7016-7028.

17 L. Tao, F. Wang, L. Lin, M. Liu and J. Sun, Magnetically separable nitrogen-doped mesoporous carbon with high adsorption capacity, J. Mater. Sci., 2016, 51, 3868-3879.

18 G. Zhu, X. Xing, J. Wang and X. Zhang, Effect of acid and hydrothermal treatments on the dye adsorption properties of biomass-derived activated carbon, J. Mater. Sci., 2017, 52, 1-13.
19 K. R. Parmar, I. Patel, S. Basha and Z. V. P. Murthy, Synthesis of acetone reduced graphene oxide $/ \mathrm{Fe}_{3} \mathrm{O}_{4}$ composite through simple and efficient chemical reduction of exfoliated graphene oxide for removal of dye from aqueous solution, J. Mater. Sci., 2014, 49, 6772-6783.

20 Y. Y. Dong, S. Liu, Y. J. Liu, L. Y. Meng and M. G. Ma, $\mathrm{Ag} @ \mathrm{Fe}_{3} \mathrm{O}_{4}$ @cellulose nanocrystals nanocomposites: microwave-assisted hydrothermal synthesis, antimicrobial properties, and good adsorption of dye solution, J. Mater. Sci., 2017, 52, 1-12.

21 Y. Li, X. Huining, M. Chen, S. Zhaoping and Y. Zhao, Absorbents based on maleic anhydride-modified cellulose fibers/diatomite for dye removal, J. Mater. Sci., 2014, 49, 6696-6704.

22 W. Song, L. Qian, B. Gao, Y. Zhu, M. Zhu, Y. Zhao, H. Ren and Z. Miao, Ionic liquid-based amphiphilic conetwork with mechanical toughness: a promising candidate for dye removal, J. Mater. Sci., 2019, 54, 6212-6226.

23 K. Yong, Y. Jia, Z. L. Wang, S. P. Yao and Z. D. Chen, Application of expanded graphite/attapulgite composite materials as electrode for treatment of textile wastewater, Appl. Clay Sci., 2009, 46, 358-362.

24 H. Jin, Z. Ji, Y. Jiao, L. Ji, L. Min, C. Xu, D. Jie, H. Pan and S. Hou, Research on removal of fluoride in aqueous solution by alumina-modified expanded graphite composite, J. Alloys Compd., 2015, 620, 361-367.

25 T. Yao, Y. Zhang, Y. Xiao, P. Zhao, G. Li, H. Yang and F. Li, The effect of environmental factors on the adsorption of lubricating oil onto expanded graphite, J. Mol. Liq., 2016, 218, 611-614.

26 S. Zhou, Z. Yan, Z. Ling, Z. Zhang and X. Fang, Modification of expanded graphite and its adsorption for hydrated salt to prepare composite PCMs, Appl. Therm. Eng., 2018, 133, S1359431117369028.

27 A. G. Bannov, A. A. Timofeeva, V. V. Shinkarev, K. D. Dyukova, A. V. Ukhina, E. A. Maksimovskii and S. I. Yusin, Synthesis and studies of properties of graphite oxide and thermally expanded graphite, Prot. Met. Phys. Chem. Surf., 2014, 50, 183-190.

28 G. Qi, W. Zhang and Y. Dai, Synthesis of Magnetic Expanded Graphite and Its Application to Remove $\mathrm{Cr}(\mathrm{VI})$ From Wastewater, Synth. React. Inorg. Met.-Org. Chem., 2014, 44, 153-160.

29 J. H. Li, L. L. Feng and Z. X. Jia, Preparation of expanded graphite with $160 \mu \mathrm{m}$ mesh of fine flake graphite, Mater. Lett., 2006, 60, 746-749.

30 H. L. Hua, Y. Wang, Y. J. Wang, S. J. Ruan, C. Zeng, T. Zhang, M. C. Zhu, Y. C. Zhang and D. X. Li, Preparation of Expanded Graphite Using Recycling Graphite Rods by Microwave Irradiation, Adv. Mater. Res., 2013, 610-613, 5.

31 S. C. Chelgani, M. Rudolph, R. Kratzsch, D. Sandmann and J. Gutzmer, A Review of Graphite Beneficiation Techniques, Miner. Process. Extr. Metall. Rev., 2016, 37, 5868.

32 I. Cameán, P. Lavela, J. L. Tirado and A. B. García, On the electrochemical performance of anthracite-based graphite 
materials as anodes in lithium-ion batteries, Fuel, 2010, 89, 986-991.

33 B. Xing, C. Zhang, Y. Cao, G. Huang, Q. Liu, C. Zhang, Z. Chen, G. Yi, L. Chen and J. Yu, Preparation of synthetic graphite from bituminous coal as anode materials for high performance lithium-ion batteries, Fuel Process. Technol., 2018, 172, 162-171.

34 G. Feng, J. Qu, Z. Zhao, Z. Quan, B. Li and J. Qiu, A green strategy for the synthesis of graphene supported Mn $3 \mathrm{O} 4$ nanocomposites from graphitized coal and their supercapacitor application, Carbon, 2014, 80, 640-650.

35 I. Cameán and A. B. Garcia, Graphite materials prepared by HTT of unburned carbon from coal combustion fly ashes: Performance as anodes in lithium-ion batteries, J. Power Sources, 2011, 196, 4816-4820.

36 Z. Quan, Z. Zhao, Y. Zhang, M. Bo, A. Zhou and J. Qiu, Graphene Sheets from Graphitized Anthracite Coal: Preparation, Decoration, and Application, Energy Fuels, 2012, 26, 5186-5192.

37 E. M. Suuberg, W. A. Peters and J. B. Howard, Product Composition and Kinetics of Lignite Pyrolysis, Industrial \& Engineering Chemistry Process Design and Development, 1977, 17, 37-46.

38 W. E. I. ChunXiu, S. H. U. I. XianYong, W. A. N. G. HengFu, C. Zhi, J. Gao and C. Wei, Investigation on the macromolecular network structure of Xianfeng lignite by a new two-step depolymerization, Fuel, 2013, 109, 49-53.

39 A. Tahmasebi, J. Yu, Y. Han, F. Yin, S. Bhattacharya and D. Stokie, Study of Chemical Structure Changes of Chinese Lignite upon Drying in Superheated Steam, Microwave, and Hot Air, Energy Fuels, 2012, 26, 3651-3660.

$40 \mathrm{~T}$. Depci, Comparison of activated carbon and iron impregnated activated carbon derived from Gölbaşı lignite to remove cyanide from water, Chem. Eng. J., 2012, 181182, 467-478.

41 G. Skodras, T. Orfanoudaki, E. Kakaras and G. P. Sakellaropoulos, Production of special activated carbon from lignite for environmental purposes, Fuel Process. Technol., 2002, 77, 75-87.

42 S. Shrestha, G. Son, S. H. Lee and T. G. Lee, Isotherm and thermodynamic studies of $\mathrm{Zn}(\mathrm{II})$ adsorption on lignite and coconut shell-based activated carbon fiber, Chemosphere, 2013, 92, 1053-1061.

43 W. Li and Y. Zhu, Structural Characteristics of Coal Vitrinite during Pyrolysis, Energy Fuels, 2014, 28, 3645-3654.
$44 \mathrm{H}$. Badenhorst, Microstructure of natural graphite flakes revealed by oxidation: Limitations of XRD and Raman techniques for crystallinity estimates, Carbon, 2014, 66, 674-690.

45 S. Huang, H. Guo, Z. Wang, G. Lei, J. Wang and X. Wei, Carbonization and graphitization of pitch applied for anode materials of high power lithium ion batteries, $J$. Solid State Electrochem., 2013, 17, 1401-1408.

46 S. Awasthi, K. Awasthi, A. K. Ghosh, S. K. Srivastava and O. N. Srivastava, Formation of single and multi-walled carbon nanotubes and graphene from Indian bituminous coal, Fuel, 2015, 147, 35-42.

47 F. Tuinstra and J. L. Koenig, Characterization of Graphite Fiber Surfaces with Raman Spectroscopy, J. Compos. Mater., 1970, 4, 492-499.

48 P. Lespade, R. Al-Jishi and M. S. Dresselhaus, Model for Raman scattering from incompletely graphitized carbons, Carbon, 1982, 20, 427-431.

49 M. Gao, Q. Ma, Q. Lin, J. Chang, W. Bao and H. Ma, Combined modification of fly ash with $\mathrm{Ca}(\mathrm{OH})_{2} / \mathrm{Na}_{2} \mathrm{FeO}_{4}$ and its adsorption of Methyl orange, Appl. Surf. Sci., 2015, 359, 323-330.

50 V. K. Dobruskin, Potential Theory of Adsorption of Nonelectrolytes from Dilute Aqueous Solutions. Benzene Adsorption, Langmuir, 1996, 12, 5606-5613.

51 K. M. Tawarah, A thermodynamic study of the association of the acid form of methyl orange with cyclodextrins, Dyes Pigm., 1992, 19, 59-67.

52 A. Siyasukh, Y. Chimupala and N. Tonanon, Preparation of magnetic hierarchical porous carbon spheres with graphitic features for high methyl orange adsorption capacity, Carbon, 2018, 134, 207-221.

53 E. Pargoletti, V. Pifferi, L. Falciola, G. Facchinetti, A. R. Depaolini, E. Davoli, M. Marelli and G. Cappelletti, A detailed investigation of $\mathrm{MnO}_{2}$ nanorods to be grown onto activated carbon. High efficiency towards aqueous methyl orange adsorption/degradation, Appl. Surf. Sci., 2018, S0169433218308560.

54 M. Wu, H. Han, L. Ni, D. Song, S. Li, T. Hu, J. Jiang and J. Chen, Preparation of 1D Hierarchical Material Mesosilica/Pal Composite and Its Performance in the Adsorption of Methyl Orange, Materials, 2018, 11, 164.

55 S. Jiao, Y. Jin, D. Qian, C. Zhu, S. Gao, D. Wang and J. Wang, Preparation of hierarchical $\beta-\mathrm{Ni}(\mathrm{OH})_{2}$ nanostructures and adsorption characterization of methyl orange dye, Solid State Sci., 2018, 79, 79-84. 